\title{
Adolescent and adult perceptions of the effects of larger size graphic health warnings on conventional and plain tobacco packs in India: A community-based cross-sectional study
}

\author{
Gaurang P. Nazar1,2, Monika Arora ${ }^{1,2}$, Vinay K. Gupta ${ }^{1}$, Tina Rawal ${ }^{1,2}$, Amit Yadav ${ }^{3}$, Nanda K. Kannuri', Surbhi \\ Shrivastava ${ }^{2}$, Nathan Grills ${ }^{5}$, Premila Webster ${ }^{6}$
}

\begin{abstract}
INTRODUCTION We studied adolescent and adult perceptions of the effects of larger size, $85 \%$ versus $40 \%$, Graphic Health Warnings (GHWs) on conventional and plain tobacco packs, in India.

METHODS A cross-sectional survey was conducted with 2121 participants (aged $\geq 13$ years), during the period 2015-16, in Delhi and Telangana, India. Four categories of GHWs on tobacco packs were shown: A - 40\% existing (April 2013-April 2016), B - 40\% new (April 2016-present), C - 85\% new, and D - plain packs ( $85 \%$ new). Regression models tested percentage differences in choice of categories for eight outcomes, adjusted for gender, area of residence, socioeconomic status, age, and tobacco use.

RESULTS Of the total 2121 participants, 1120 were from Delhi, 1001 from Telangana, $50 \%$ were males, $62 \%$ were urban residents, $12 \%$ were adolescents, and $72 \%$ had never used tobacco. Among packs shown, the majority of participants perceived the $85 \%$ size GHWs more effective than the $40 \%$ size GHWs across all outcomes. The perceived increase in noticeability of $\mathrm{GHW}$ s was $45 \%$ for category $\mathrm{C}(\mathrm{p}<0.05)$ and $43.5 \%$ for category D $(\mathrm{p}<0.05)$ versus category B. In Delhi, participants perceived plain packs to be most effective in motivating quitting, preventing initiation and conveying the health message. In Telangana, adolescents believed GHWs on plain packs were most noticeable, most effective for quitting and preventing initiation.

CONCLUSIONS The larger size $85 \%$ GHWs were perceived to be more effective in increasing noticeability of warnings, motivating cessation, preventing initiation, and conveying the intended health message. Support for plain packaging was higher in Delhi and among adolescents in Telangana.
\end{abstract}

AFFILIATION
1 Health Promotion Division,
Public Health Foundation of
India, Gurugram, India
2 HRIDAY, New Delhi, India
3 Center for Tobacco Control
Research and Education,
University of California, San
Francisco, United States
4 Indian Institute of Public
Health, Hyderabad, India
5 Nossal Institute for
Global Health, University
of Melbourne, Melbourne,
Australia
6 Nuffield Department of
Population Health, University
of Oxford, Oxford, United
Kingdom

CORRESPONDENCE TO Gaurang P. Nazar. Health Promotion Division, Public Health Foundation of India, Plot No. 47, Institutional Area, Sector 44, Gurugram, Haryana 122002, India. E-mail: gaurang.nazar@phfi.org ORCID ID: https://orcid. org/0000-0002-7025-2946

KEYWORDS

perception, tobacco, plain packaging, graphic health warnings, India

Received: 10 May 2019 Revised: 7 July 2019 Accepted: 12 July 2019

\section{INTRODUCTION}

Tobacco use leads to more than 1.3 million deaths annually in India ${ }^{1,2}$, and this is expected to rise to 1.5 million by $2020^{3}$. Adolescents are particularly vulnerable to tobacco industry advertising and promotion, which can lead to initiation of tobacco use $^{4}$. The mean age of initiation of tobacco in India is 18.9 years $^{1,2}$. Therefore, adolescents represent an 
important target for tobacco prevention and control efforts. Tobacco control in India is complicated by the range of tobacco products and packs available. The Government of India (GoI) implemented the Cigarettes and Other Tobacco Products Act (COTPA) in $2003^{5}$. Section 7 of COTPA mandates the display of graphic health warnings (GHWs) on all tobacco packs $^{5,6}$. GHWs covering $40 \%$ of the principal display area (PDA) [front panel] of the pack were introduced in India from 31 May 2009, and were rotated in the years 2011, 2013 and $2016^{7}$. In 2014, the GoI notified for larger, field-tested GHWs (covering top $85 \%$ of the PDA [front and back] of tobacco packs), which were implemented from April 2016 $6^{6,8}$. The Karnataka High Court struck down the 2014 notification in December 2017 but the notification was upheld by the Supreme Court of India in January 2018 $8^{9-11}$. However, legal challenges continue to threaten the implementation of large GHWs in India.

The implementation of plain packaging with large GHWs is the next step towards a comprehensive tobacco control policy, and specifically, to prevent tobacco use among adolescents. Plain packaging of tobacco products was implemented as a demandreduction strategy to protect young people initially in Australia in $2012^{12}$, followed by France, the United Kingdom, New Zealand, Norway, and Ireland ${ }^{12}$. Australian research suggests that plain packaging has accelerated the decline in smoking prevalence, reduced the appeal of tobacco packs as well as the ability of packs to mislead consumers, and enhanced the effectiveness of $\mathrm{GHW} \mathrm{s}^{13}$. Plain packaging in India would also be expected to make tobacco packs less attractive. At present, COTPA (2003) $)^{5}$ allows on-pack advertising, which makes tobacco packs attractive. An India-Australia High-level Taskforce was set up in India to explore the feasibility of plain packaging in 2012. The preliminary research evidence ${ }^{14,15}$ led to the introduction of a private members' bill on plain packaging in the Indian Parliament ${ }^{16}$.

In countries that have implemented plain packaging, public consultations were undertaken to obtain the views of the public and experts on the concept of plain packaging ${ }^{17}$. Such public consultations and research on perceptions of the impact of plain tobacco packaging and larger GHWs (from $40 \%$ to $85 \%$ ) on various tobacco-use outcomes have not been undertaken in India.
The objective of this study was to assess perceptions of Indian adolescents (aged 13-17 years) and adults ( $\geq 18$ years) of the effect of the $2016 \mathrm{GHWs}$ and their increased size from $40 \%$ to $85 \%$ on conventional tobacco packs compared with plain packs.

The pre-specified hypothesis was that increasing the size of GHWs to $85 \%$ and placing them on plain packaging would increase the noticeability of the warnings by at least $5 \%$ compared with $40 \%$ size warnings placed on conventional packs.

\section{METHODS}

\section{Study design}

This community-based, cross-sectional study was conducted in February-March 2016, using an interviewer-administered questionnaire during individual face-to-face interviews with study participants. The study was conducted after the notification (October 2014) but before the implementation (April 2016) of new 85\% sized GHWs in India. Hence, the $40 \%$ size GHWs that were in force between April 2013 and April 2016 were imprinted on the tobacco packs when this study was conducted. Therefore, these packs were included in this study. Conventional tobacco packs with $40 \%$ sized existing (April 2013 - April 2016) warnings (pack category A), dummy tobacco packs with 40\% new (April 2016 present) warnings (pack category B), dummy tobacco packs with $85 \%$ new warnings on conventional packs (pack category G) and dummy tobacco packs with $85 \%$ new warnings on plain packs (pack category D) were shown (Figure 1) to participants to elicit responses to questions asked by the interviewer using the questionnaire.

\section{Study setting}

The study was conducted in communities of New Delhi (urban) and Najafgarh (rural) districts in the National Capital Territory (NCT) of Delhi, and in Hyderabad (urban) and Ranga Reddy (rural) districts in the state of Telangana. The NCT of Delhi represented north India and the state of Telangana represented south India.

\section{Participants}

The sample size was estimated for the difference between two proportions, based on the reported noticeability of GHWs on tobacco packs as the 


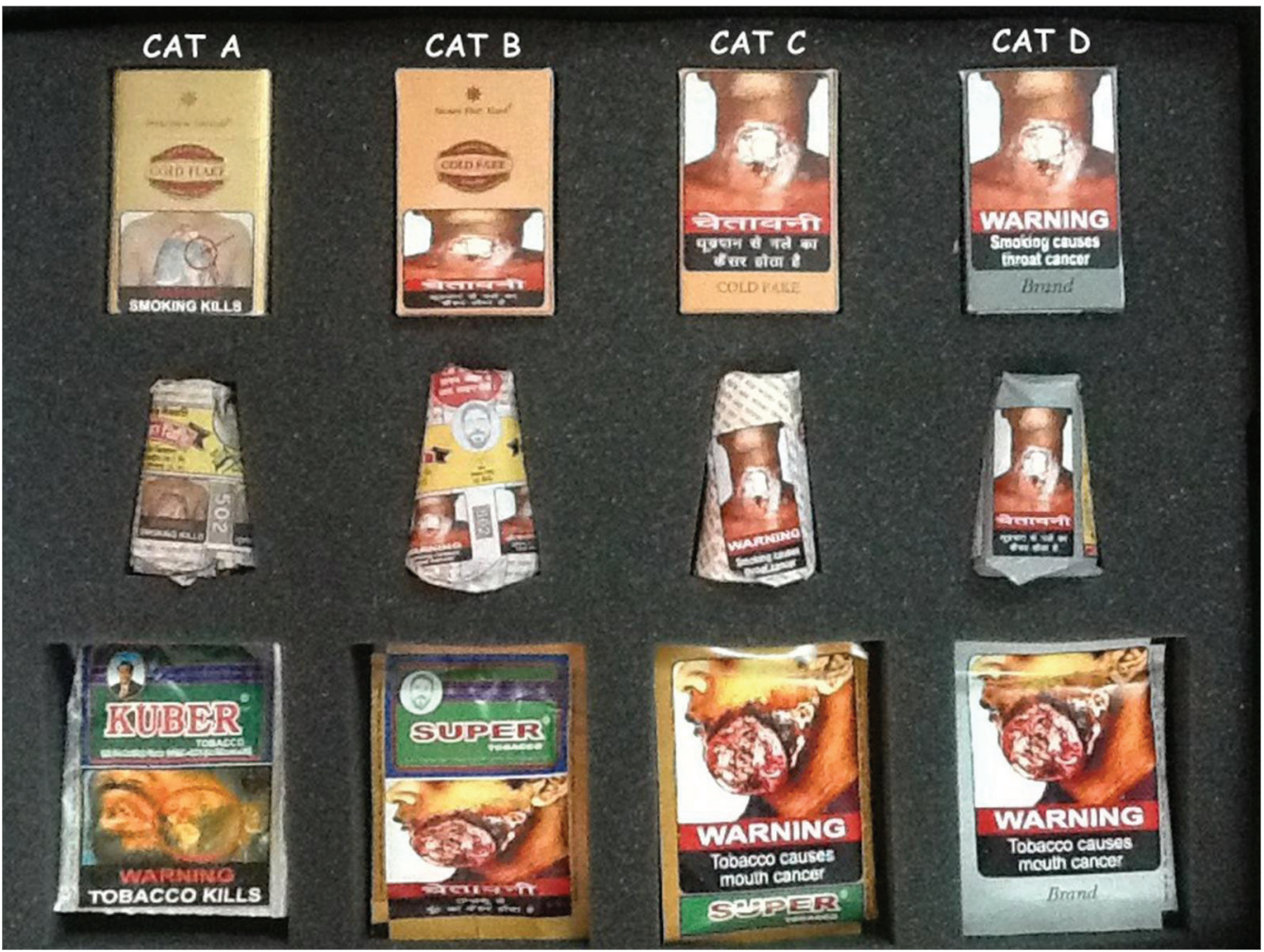

A - 40\% existing (April 2013 - April 2016) GHW on conventional cigarette pack, bidi pack and smokeless tobacco sachet. B - 40\% new (April 2016 - present) GHW on conventional cigarette pack, bidi pack and smokeless tobacco sachet. C - 85\% new (April 2016 - present) GHW on conventional cigarette pack, bidi pack and smokeless tobacco sachet. D - 85\% new (April 2016 - present) GHW on plain cigarette pack, bidi pack and smokeless tobacco sachet.

outcome, which was considered to be $28 \%$ based on a previous Indian study ${ }^{15}$. Assuming a $5 \%$ increase in the proportion of the population that will notice (reportedly observe) warnings after increasing the size from $40 \%$ to $85 \%$, and placing these on plain packs, the minimum estimated sample size was 1326 . The sample size was finalised as 2000 (1000 in each state). The sample was further divided between rural and urban areas and age groups based on 2011 census population ratio $^{18}$.

Participants aged $\geq 13$ years were selected, consistent with the nationally representative surveys: Global Youth Tobacco Survey (GYTS) ${ }^{19}$, and Global Adult Tobacco Survey (GATS) ${ }^{1}$. Also, consistent with the sampling method used by GYTS and GATS, a two-stage sampling scheme was used. The primary sampling units (PSU) were villages in the rural area and wards in urban areas. These were selected using probability proportional to size. Twenty households from each PSU and one participant from each household were selected. As no accurate household lists were available in villages, each village was identified on Google maps and geographically divided into two equal parts. From each part, 10 males and 10 females were selected. This was based on the Expanded Program on Immunization method used by WHO and UNICEF in the absence of suitable sampling frame ${ }^{20}$. Similarly, wards in the urban area were divided into two parts and 10 houses were selected from each part through a systematic sampling technique. The overall response rate was $70 \%$.

\section{The questionnaire}

The questionnaire was developed in consultation with experts in tobacco control in India and Australia, building on the previous feasibility study conducted by the India-Australia High-level Taskforce ${ }^{15}$. It included components from previously validated 
tools used for testing perceptions of the effects of modified packaging with different sizes of GHWs in Australia $^{21}$, and in Canada with adults ${ }^{22}$, and young people $^{23}$. Data were collected on demography, tobacco use behaviour, perceived noticeability of GHWs, the appeal of tobacco packs, and perceived effectiveness of GHWs on various indicators related to tobacco use. Participants were shown four categories of tobacco packs simultaneously ${ }^{21}$ to elicit their responses during the survey (Figure 1).

The questionnaire was developed in English, translated to Hindi (Delhi) and Telugu (Telangana) and then back-translated to English. After piloting the questionnaire with 100 participants in Delhi and Hyderabad to test face and content validity, appropriate changes were incorporated. Written informed consent was obtained from all adult participants and informed assent was obtained from adolescents, after obtaining their parents'/guardians' consent. Ethics approval for the study was obtained from the Institutional Ethics Committee at Public Health Foundation of India (TRC-IEC-252/15).

\section{Outcome variables}

The outcome variables were participant perceptions of the four categories of tobacco product packs shown to them. Participants were asked which of the four categories they thought would:

- Be most effective in motivating tobacco users to quit;

- Be most effective in preventing initiation of tobacco products among non-users;

- Be most likely to make participants think that the health risks of tobacco use are extremely serious;

- Make GHWs stand out the most/make them most noticeable;

- Be most effective in conveying the GHW message so that it is easily understood;

- Be most likely to attract an adult to use the product;

- Be most likely to attract children and adolescents to use the product.

Support for GoI-introduced larger GHW, from $40 \%$ to $85 \%$, was also assessed.

\section{Data analysis}

The socioeconomic status (SES) variable combined education, occupation and income through Kuppuswamy's SES scale criteria ${ }^{24}$. Tobacco use status was categorized as user (current- and everuser) and non-user. Age groups were dichotomized as adolescents (aged 13-17 years) and adults (aged $\geq 18$ years). Stata $v \cdot 13 \cdot 1$ (StataCorp LP, TX) was used for data analyses. Frequency distributions and percentages were provided for sociodemographic characteristics and responses about perceptions of the four categories of tobacco packs. Associations of perceptions between age groups, area (rural/urban), gender, SES groups and tobacco use status were obtained using Pearson's chi-squared test or Fisher's exact test, as appropriate (Supplementary Tables S2S6). We used linear regression to test the differences in percentages between the choice of tobacco packs ( $\mathrm{C}$ and $\mathrm{B} ; \mathrm{D}$ and $\mathrm{B} ; \mathrm{D}$ and $\mathrm{C}$ ) for each outcome, after adjusting for covariates significantly associated with the outcomes. Comparisons with pack category A are presented in Supplementary files (Tables S7-S9) and not discussed in great detail in this paper since they became non-existent in India soon after the study and hence irrelevant for tobacco control policy.

\section{RESULTS}

\section{Demographic characteristics}

The study included 2121 participants (Delhi 1120, Telangana 1001) (Table 1). Fifty per cent of participants were males, while $12.5 \%$ of participants came from the rural area in Delhi compared with 66\% in Telangana. About $12 \%$ of the participants were aged 13-17 years, just under 50\% were aged $25-44$ years and about one-fifth were aged $\geq 45$ years. Half of the participants were in the low SES (lower and upper lower) category, $46 \%$ belonged to middle SES (lower middle and upper middle) category and only $4 \%$ belonged to upper SES category. A quarter of participants currently used tobacco while $72 \%$ had never used tobacco.

\section{Table 1. Sociodemographic characteristics of} respondents

\begin{tabular}{|c|c|c|c|c|c|c|}
\hline & \multicolumn{2}{|c|}{$\begin{array}{c}\text { Delli } \\
(n-1120)\end{array}$} & \multicolumn{2}{|c|}{$\begin{array}{c}\text { Telangana } \\
(n-1001)\end{array}$} & \multicolumn{2}{|c|}{$\begin{array}{c}\text { Total } \\
\text { (N 2121) }\end{array}$} \\
\hline & $\mathbf{N}$ & $\%$ & $\mathbf{N}$ & $\%$ & $\mathbf{N}$ & $\%$ \\
\hline \multicolumn{7}{|l|}{ Gender } \\
\hline Male & 560 & 50 & 500 & 50 & 1060 & 50 \\
\hline Female & 560 & 50 & 501 & 50 & 1061 & 50 \\
\hline
\end{tabular}


Table 1. Continued

\begin{tabular}{|c|c|c|c|c|c|c|}
\hline & \multicolumn{2}{|c|}{$\begin{array}{c}\text { Dellhi } \\
(\mathrm{n}-1120)\end{array}$} & \multicolumn{2}{|c|}{$\begin{array}{c}\text { Telangana } \\
\text { (n-1001) }\end{array}$} & \multicolumn{2}{|c|}{$\begin{array}{c}\text { Total } \\
\text { (N 2121) }\end{array}$} \\
\hline & $\mathbf{N}$ & $\%$ & $\mathbf{N}$ & $\%$ & $\mathbf{N}$ & $\%$ \\
\hline \multicolumn{7}{|l|}{ Area } \\
\hline Urban & 980 & 87.5 & 341 & 34 & 1321 & 62 \\
\hline Rural & 140 & 12.5 & 660 & 66 & 800 & 38 \\
\hline \multicolumn{7}{|c|}{ Age group (years) } \\
\hline $13-17$ & 147 & 13 & 100 & 10 & 247 & 12 \\
\hline $18-24$ & 183 & 16 & 207 & 21 & 390 & 18 \\
\hline $25-44$ & 528 & 47 & 501 & 50 & 1029 & 49 \\
\hline$\geq 45$ & 262 & 23 & 193 & 19 & 455 & 21 \\
\hline \multicolumn{7}{|c|}{$\begin{array}{l}\text { Socioeconomic } \\
\text { status (SES)* }\end{array}$} \\
\hline Lower & 16 & 2 & 49 & 5 & 65 & 3 \\
\hline Upper lower & 444 & 40 & 551 & 55 & 995 & 47 \\
\hline Lower middle & 376 & 34 & 254 & 25 & 630 & 30 \\
\hline Upper middle & 222 & 20 & 105 & 11 & 327 & 16 \\
\hline Upper & 41 & 4 & 41 & 4 & 82 & 4 \\
\hline \multicolumn{7}{|l|}{$\begin{array}{l}\text { Tobacco use } \\
\text { status** }\end{array}$} \\
\hline Current user & 252 & 23 & 277 & 28 & 529 & 25 \\
\hline Ever user & 35 & 3 & 18 & 2 & 53 & 2.50 \\
\hline Never user & 833 & 74 & 704 & 70 & 1537 & 72.5 \\
\hline
\end{tabular}

Participant perceptions of the effect of pack categories

Adjusted regression results suggest that the percentage of participants who perceived increased noticeability of GHWs was significantly higher for the $85 \%$ size GHWs on conventional packs (category C) as well as for $85 \%$ size $\mathrm{GHW}$ s on plain packs (category D), compared with $40 \%$ size GHWs on conventional packs (category B) (Table 2). The reported increase in perceived noticeability was $45 \%$ for category C compared with category B $(\mathrm{p}<0.05)$, and $43.5 \%$ for category D compared with category B $(p<0.05)$. The difference in perceived noticeability was not significant between category D compared with category C. In Delhi, the reported increase in perceived noticeability was higher for category $\mathrm{D}$ than category C, although the difference between D and C was not significant (Table 3 ).

Category D packs were perceived to be more effective than category $\mathrm{C}$ in preventing initiation among non-users when each was compared with category B (50\% vs 39\%) (Table 2). However, the difference between categories $\mathrm{D}$ and $\mathrm{C}$ was not significant. In Delhi, category D was perceived to be significantly more effective for this outcome

Table 2. Percentage difference between the choice of tobacco pack category (Overall, $n=2121$ )

\begin{tabular}{|c|c|c|c|c|c|c|}
\hline & \multicolumn{2}{|c|}{ Between pack C and B } & \multicolumn{2}{|c|}{ Between pack D and B } & \multicolumn{2}{|c|}{ Between pack D and C } \\
\hline & $\begin{array}{l}\text { Unadjusted \% } \\
\text { difference } \\
\left(95^{\circ} \% \text { CI }\right)\end{array}$ & $\begin{array}{l}\text { Adjusted } \% \\
\text { difference } \\
\left(95^{\circ} \circ \mathrm{CI}\right)^{*}\end{array}$ & $\begin{array}{l}\text { Unadjusted } \% \\
\text { difference } \\
(95 \% \mathrm{CI})\end{array}$ & $\begin{array}{l}\text { Adjusted \% } \\
\text { difference } \\
\left(95^{\circ} \% \text { CI }\right)^{*}\end{array}$ & $\begin{array}{l}\text { Unadjusted \% } \\
\text { difference } \\
(95 \% \text { CI })\end{array}$ & $\begin{array}{l}\text { Adjusted \% } \\
\text { difference } \\
\left(95^{\circ} \% \text { CI }\right)^{*}\end{array}$ \\
\hline $\begin{array}{l}\text { 1. Most effective } \\
\text { in motivating } \\
\text { tobacco users to } \\
\text { quit }\end{array}$ & $\begin{array}{c}49.34 \\
(46.99-51.69)\end{array}$ & $\begin{array}{c}49.38 \\
(47.05-51.72)\end{array}$ & $\begin{array}{c}40.94 \\
(38.62-43.26)\end{array}$ & $\begin{array}{c}41.03 \\
(38.71-43.34)\end{array}$ & $\begin{array}{c}-8.40 \\
(-12.55--4.24)\end{array}$ & $\begin{array}{c}-8.36 \\
(-12.50--4.21)\end{array}$ \\
\hline $\begin{array}{l}\text { 2. Most effective } \\
\text { in preventing } \\
\text { initiation of } \\
\text { tobacco use }\end{array}$ & $\begin{array}{c}39.17 \\
(36.90-41.44)\end{array}$ & $\begin{array}{c}39.19 \\
(36.91-41.47)\end{array}$ & $\begin{array}{c}49.98 \\
(47.66-52.29)\end{array}$ & $\begin{array}{c}50.02 \\
(47.69-52.35)\end{array}$ & $\begin{array}{c}10.81 \\
(6.71-14.91)\end{array}$ & $\begin{array}{c}10.83 \\
(6.71-14.96)\end{array}$ \\
\hline $\begin{array}{l}\text { 3. Most likely to } \\
\text { make you think } \\
\text { that health risks } \\
\text { of tobacco are } \\
\text { extremely serious }\end{array}$ & $\begin{array}{c}46.22 \\
(43.87-48.58)\end{array}$ & $\begin{array}{c}46.27 \\
(43.92-48.62)\end{array}$ & $\begin{array}{c}43.91 \\
(41.56-46.25)\end{array}$ & $\begin{array}{c}43.86 \\
(41.52-46.20)\end{array}$ & $\begin{array}{c}-2.31 \\
(-6.48-1.86)\end{array}$ & $\begin{array}{c}-2.41 \\
(-6.58-1.76)\end{array}$ \\
\hline $\begin{array}{l}\text { 4. GHWs are most } \\
\text { noticeable }\end{array}$ & $\begin{array}{c}45.25 \\
(42.90-47.61)\end{array}$ & $\begin{array}{c}45.26 \\
(42.91-47.60)\end{array}$ & $\begin{array}{c}43.51 \\
(41.16-45.86)\end{array}$ & $\begin{array}{c}43.51 \\
(41.16-45.86)\end{array}$ & $\begin{array}{c}-1.74 \\
(-5.89-2.40)\end{array}$ & $\begin{array}{c}-1.75 \\
(-5.89-2.39)\end{array}$ \\
\hline $\begin{array}{l}\text { 5. Message } \\
\text { conveyed by the } \\
\mathrm{GHW} \text { is easiest to }\end{array}$ & $\begin{array}{c}50.26 \\
(47.94-52.57)\end{array}$ & $\begin{array}{c}50.26 \\
(47.95-52.57)\end{array}$ & $\begin{array}{c}41.95 \\
(39.66-44.24)\end{array}$ & $\begin{array}{c}41.95 \\
(39.67-44.24)\end{array}$ & $\begin{array}{c}-8.30 \\
(-12.48--4.13)\end{array}$ & $\begin{array}{c}-8.30 \\
(-12.47--4.13)\end{array}$ \\
\hline
\end{tabular}


Table 2. continued

\begin{tabular}{|c|c|c|c|c|c|c|}
\hline & \multicolumn{2}{|c|}{ Between pack $C$ and B } & \multicolumn{2}{|c|}{ Between pack D and B } & \multicolumn{2}{|c|}{ Between pack D and C } \\
\hline & $\begin{array}{l}\text { Unadjusted \% } \\
\text { difference } \\
\left(95^{\circ} \% \text { CI }\right)\end{array}$ & $\begin{array}{l}\text { Adjusted \% } \\
\text { difference } \\
(95 \% \text { CI) }\end{array}$ & $\begin{array}{l}\text { Unadjusted \% } \\
\text { difference } \\
(95 \% \mathrm{CI})\end{array}$ & $\begin{array}{l}\text { Adjusted "\% } \\
\text { difference } \\
\left(95^{\circ} \% \mathrm{CI}\right)^{*}\end{array}$ & $\begin{array}{l}\text { Unadjusted \% } \\
\text { difference } \\
\left(95^{\circ} \% \text { CI }\right)\end{array}$ & $\begin{array}{l}\text { Adfusted } \% \\
\text { difference } \\
\left(95^{\circ} \% \mathrm{CI}\right)^{*}\end{array}$ \\
\hline $\begin{array}{l}\text { 6. Most likely to } \\
\text { lure adults into } \\
\text { using tobacco } \\
\text { products }\end{array}$ & $\begin{array}{c}-13.46 \\
(-15.54--11.37)\end{array}$ & $\begin{array}{c}-13.21 \\
(-15.27--11.14)\end{array}$ & $\begin{array}{c}-15.67 \\
(-17.63--13.72)\end{array}$ & $\begin{array}{c}-15.45 \\
(-17.37--13.53)\end{array}$ & $\begin{array}{c}-2.22 \\
(-3.57--0.87)\end{array}$ & $\begin{array}{c}-2.24 \\
(-3.60--0.88)\end{array}$ \\
\hline $\begin{array}{l}\text { 7. Most likely to } \\
\text { lure children and } \\
\text { adolescents into } \\
\text { using tobacco } \\
\text { products }\end{array}$ & $\begin{array}{c}-12.07 \\
(-14.17--9.98)\end{array}$ & $\begin{array}{c}-11.72 \\
(-13.80--9.64)\end{array}$ & $\begin{array}{c}-14.00 \\
(-15.99--12.02)\end{array}$ & $\begin{array}{c}-13.68 \\
(-15.64--11.71)\end{array}$ & $\begin{array}{c}-1.93 \\
(-3.38--0.48)\end{array}$ & $\begin{array}{c}-1.95 \\
(-3.42--0.49)\end{array}$ \\
\hline $\begin{array}{l}\text { 8. Support for } \\
\text { pack category }\end{array}$ & $\begin{array}{c}49.98 \\
(47.55-52.40)\end{array}$ & $\begin{array}{c}50.17 \\
(47.82-52.52)\end{array}$ & $\begin{array}{c}39.12 \\
(36.75-41.50)\end{array}$ & $\begin{array}{c}39.10 \\
(36.76-41.44)\end{array}$ & $\begin{array}{c}-10.85 \\
(-15.01--6.70)\end{array}$ & $\begin{array}{c}-11.07 \\
(-15.11--7.03)\end{array}$ \\
\hline
\end{tabular}

*Linear regression model adjusted for covariates that were significantly associated with the outcome. The covariates were gender (female/male), area (rural/urban), socioeconomic status (low/middle/high), age groups (13-17/18-24/25-44/ $\geq 45$ years) and tobacco use (never user/user). 01-SES excluded; 02-age group excluded; 03-gender/SES excluded; Q4-gender/SES/tobacco use excluded; $05-\mathrm{SES} /$ tobacco use/age group excluded; $06,7,8$-age group and tobacco use excluded. Bold numbers indicate significance ( $<<0.05$ ).

Table 3. Percentage difference between the choice of tobacco pack category (Delhi, $\mathbf{n = 1 1 2 0 )}$

\begin{tabular}{|c|c|c|c|c|c|c|}
\hline & \multicolumn{2}{|c|}{ Between pack $C$ and B } & \multicolumn{2}{|c|}{ Between pack D and B } & \multicolumn{2}{|c|}{ Between pack D and C } \\
\hline & $\begin{array}{l}\text { Unadjusted } \% \\
\text { difference } \\
(95 \% \text { CI) }\end{array}$ & $\begin{array}{l}\text { Adjusted \% } \\
\text { diflerence } \\
(95 \% \text { CI) }\end{array}$ & $\begin{array}{l}\text { Unadjusted \% } \\
\text { difference } \\
\left(95^{\circ} \% \mathrm{CI}\right)\end{array}$ & $\begin{array}{l}\text { Adjusted \% } \\
\text { dilference } \\
\left(95^{\circ} \% \mathrm{CI}\right)^{*}\end{array}$ & $\begin{array}{l}\text { Unadjusted \% } \\
\text { diflerence } \\
\left(95^{\circ} \% \mathrm{CI}\right)\end{array}$ & $\begin{array}{l}\text { Adjusted \% } \\
\text { difference } \\
(95 \% \text { CI) }\end{array}$ \\
\hline $\begin{array}{l}\text { 1. Most effective in } \\
\text { motivating tobacco } \\
\text { users to quit }\end{array}$ & $\begin{array}{c}37.86 \\
(34.57-41.14)\end{array}$ & $\begin{array}{c}37.86 \\
(34.57-41.14)\end{array}$ & $\begin{array}{c}47.77 \\
(44.41-51.13)\end{array}$ & $\begin{array}{c}47.77 \\
(44.43-51.11)\end{array}$ & $\begin{array}{c}9.91 \\
(4.27-15.55)\end{array}$ & $\begin{array}{c}9.91 \\
(4.29-15.53)\end{array}$ \\
\hline $\begin{array}{l}\text { 2. Most effective in } \\
\text { preventing initiation } \\
\text { of tobacco use }\end{array}$ & $\begin{array}{c}37.41 \\
(34.20-40.62)\end{array}$ & $\begin{array}{c}37.21 \\
(33.98-40.45)\end{array}$ & $\begin{array}{c}45.80 \\
(42.51-49.09)\end{array}$ & $\begin{array}{c}45.80 \\
(42.51-49.09)\end{array}$ & $\begin{array}{c}8.39 \\
(2.85-13.93)\end{array}$ & $\begin{array}{c}8.55 \\
(3.00-14.10)\end{array}$ \\
\hline $\begin{array}{l}\text { 3. Most likely to make } \\
\text { you think that health } \\
\text { risks of tobacco are } \\
\text { extremely serious }\end{array}$ & $\begin{array}{c}42.41 \\
(39.02-45.80)\end{array}$ & $\begin{array}{c}42.41 \\
(39.02-45.80)\end{array}$ & $\begin{array}{c}42.05 \\
(38.67-45.44)\end{array}$ & $\begin{array}{c}42.05 \\
(38.70-45.40)\end{array}$ & $\begin{array}{c}-0.36 \\
(-6.02-5.31)\end{array}$ & $\begin{array}{c}-0.36 \\
(-6.02-5.31)\end{array}$ \\
\hline $\begin{array}{l}\text { 4. GHWs are most } \\
\text { noticeable }\end{array}$ & $\begin{array}{c}37.32 \\
(33.95-40.69)\end{array}$ & $\begin{array}{c}37.32 \\
(33.95-40.69)\end{array}$ & $\begin{array}{c}43.48 \\
(40.05-46.91)\end{array}$ & $\begin{array}{c}43.48 \\
(40.05-46.91)\end{array}$ & $\begin{array}{c}6.16 \\
(0.59-11.73)\end{array}$ & $\begin{array}{c}6.16 \\
(0.59-11.73)\end{array}$ \\
\hline $\begin{array}{l}\text { 5. Message conveyed } \\
\text { by the GHW is easiest } \\
\text { to understand }\end{array}$ & $\begin{array}{c}42.89 \\
(39.59-46.20)\end{array}$ & $\begin{array}{c}42.88 \\
(39.59-46.17)\end{array}$ & $\begin{array}{c}44.59 \\
(41.28-47.90)\end{array}$ & $\begin{array}{c}44.62 \\
(41.36-47.88)\end{array}$ & $\begin{array}{c}1.70 \\
(-4.00-7.4)\end{array}$ & $\begin{array}{c}1.73 \\
(-3.90-7.38)\end{array}$ \\
\hline $\begin{array}{l}\text { 6. Most likely to lure } \\
\text { adults into using } \\
\text { tobacco products }\end{array}$ & $\begin{array}{c}-23.61 \\
(-26.99--20.23)\end{array}$ & $\begin{array}{c}-23.31 \\
(-26.70--19.92)\end{array}$ & $\begin{array}{c}-25.22 \\
(-28.47--21.97)\end{array}$ & $\begin{array}{c}-24.94 \\
(-28.19--21.69)\end{array}$ & $\begin{array}{c}-1.61 \\
(-3.76-0.54)\end{array}$ & $\begin{array}{c}-1.63 \\
(-3.82-0.56)\end{array}$ \\
\hline $\begin{array}{l}\text { 7. Most likely to } \\
\text { lure children and } \\
\text { adolescents into using } \\
\text { tobacco products }\end{array}$ & $\begin{array}{c}-22.68 \\
(-25.84--19.51)\end{array}$ & $\begin{array}{c}-22.20 \\
(-25.38--19.02)\end{array}$ & $\begin{array}{c}-21.70 \\
(-24.94--18.45)\end{array}$ & $\begin{array}{c}-21.20 \\
(-24.44--17.96)\end{array}$ & $\begin{array}{c}0.98 \\
(-1.10-3.06)\end{array}$ & $\begin{array}{c}1.00 \\
(-1.12-3.12)\end{array}$ \\
\hline $\begin{array}{l}\text { 8. Support for pack } \\
\text { category }\end{array}$ & $\begin{array}{c}28.21 \\
(24.80-31.62)\end{array}$ & $\begin{array}{c}28.21 \\
(24.77-31.64)\end{array}$ & $\begin{array}{c}51.43 \\
(47.79-55.07)\end{array}$ & $\begin{array}{c}51.59 \\
(47.93-55.26)\end{array}$ & $\begin{array}{c}23.21 \\
(17.72-28.71)^{*}\end{array}$ & $\begin{array}{c}23.38 \\
(17.84-28.92)\end{array}$ \\
\hline
\end{tabular}

*Linear regression model adjusted for covariates that were significantly associated with the outcome. The covariates were gender (female/male), area (rural/urban), socioeconomic status (low/middle/high), age groups (13-17/18-24/25-44/ $\geq 45$ years) and tobacco use (never user/user). 01-SES excluded; 02-age group excluded; 03-gender/SES excluded; Q4-gender/SES/tobacco use excluded; 05-SES/tobacco use/age group excluded; 06, 7, 8-age group and tobacco use excluded. Bold numbers indicate significance ( $<<0.05$ ). 
than category $\mathrm{C}$ by $8 \%$ (Table 3 ). Further, in Delhi, category $\mathrm{D}$ was perceived to be significantly more effective than category $\mathrm{C}$ for motivating tobacco users to quit, by almost $10 \%$.

Category B packs compared with category C, were perceived to be more likely to lure adults as well as adolescents into using tobacco products, than category B compared with D (Table 2). However, no significant differences were observed between categories D and $\mathrm{C}$ for these outcomes. The results were almost consistent in Delhi and Telangana (Tables 3 and 4).

Overall support was significantly higher for category $\mathrm{C}$ packs compared with D by $11 \%$ (Table 2 ) and in Telangana by $49 \%$ (Table 4 ). In Delhi, support was higher for category D compared with C by a significant $23 \%$ difference (Table 3 ).

When category A packs were compared with category B packs, the percentage differences in the choice of packs were not significantly different for most outcomes except that the participants, overall, and in both states, perceived that category A packs would be more likely to lure adults as well as children and adolescents into using tobacco products than category B packs ( $\mathrm{p}<0.05$ for both outcomes, overall and in both states). Across all outcomes, the participants perceived category A packs to be significantly less effective than category $\mathrm{C}$ and category D packs.

Table 4. Percentage difference between the choice of tobacco pack category (Telangana, $n=1001$ )

\begin{tabular}{|c|c|c|c|c|c|c|}
\hline & \multicolumn{2}{|c|}{ Between pack C and B } & \multicolumn{2}{|c|}{ Between pack D and B } & \multicolumn{2}{|c|}{ Between pack D and C } \\
\hline & $\begin{array}{l}\text { Unadjusted "\% } \\
\text { difference } \\
\left(95^{\circ} \% \mathrm{CI}\right)\end{array}$ & $\begin{array}{l}\text { Adjusted \% } \\
\text { diflerence } \\
\left(95^{\circ} \% \mathrm{CI}\right)^{*}\end{array}$ & $\begin{array}{l}\text { Unadjusted } \% \\
\text { diflerence } \\
\left(95^{\circ} \% \mathrm{CI}\right)\end{array}$ & $\begin{array}{l}\text { Adjusted "o } \\
\text { diflerence } \\
\left(95^{\circ} \% \mathrm{CI}\right)^{*}\end{array}$ & $\begin{array}{l}\text { Unadjusted \% } \\
\text { difference } \\
(95 \% \text { CI })\end{array}$ & $\begin{array}{l}\text { Adjusted "o } \\
\text { difference } \\
\left(95^{\circ} \% \mathrm{CI}\right)^{*}\end{array}$ \\
\hline $\begin{array}{l}\text { 1. Most effective } \\
\text { in motivating } \\
\text { tobacco users to } \\
\text { quit }\end{array}$ & $\begin{array}{c}62.20 \\
(59.01-65.38)\end{array}$ & $\begin{array}{c}62.34 \\
(59.21-65.47)\end{array}$ & $\begin{array}{c}33.30 \\
(30.19-36.40)\end{array}$ & $\begin{array}{c}33.45 \\
(30.39-36.52)\end{array}$ & $\begin{array}{c}-28.90 \\
(-34.79--23.01)\end{array}$ & $\begin{array}{c}-28.88 \\
(-34.70--23.07)\end{array}$ \\
\hline $\begin{array}{l}\text { 2. Most effective } \\
\text { in preventing } \\
\text { initiation of } \\
\text { tobacco use }\end{array}$ & $\begin{array}{c}41.14 \\
(37.94-44.34)\end{array}$ & $\begin{array}{c}41.35 \\
(38.18-44.51)\end{array}$ & $\begin{array}{c}54.65 \\
(51.41-57.89)\end{array}$ & $\begin{array}{c}54.73 \\
(51.52-57.93)\end{array}$ & $\begin{array}{c}13.51 \\
(7.41-19.61)\end{array}$ & $\begin{array}{c}13.38 \\
(7.33-19.43)\end{array}$ \\
\hline $\begin{array}{l}\text { 3. Most likely to } \\
\text { make you think } \\
\text { that health risks } \\
\text { of tobacco are } \\
\text { extremely serious }\end{array}$ & $\begin{array}{c}50.50 \\
(47.27-53.73)\end{array}$ & $\begin{array}{c}50.61 \\
(47.40-53.81)\end{array}$ & $\begin{array}{c}45.99 \\
(42.77-49.21)\end{array}$ & $\begin{array}{c}45.88 \\
(42.66-49.10)\end{array}$ & $\begin{array}{c}-4.51 \\
(-10.67-16.52)\end{array}$ & $\begin{array}{c}-4.72 \\
(-10.87-1.42)\end{array}$ \\
\hline $\begin{array}{l}\text { 4. GHWs are most } \\
\text { noticeable }\end{array}$ & $\begin{array}{c}54.15 \\
(50.97-57.33)\end{array}$ & $\begin{array}{c}54.15 \\
(50.97-57.33)\end{array}$ & $\begin{array}{c}43.54 \\
(40.38-46.71)\end{array}$ & $\begin{array}{c}43.54 \\
(40.38-46.71)\end{array}$ & $\begin{array}{c}-10.61 \\
(-16.76--4.46)\end{array}$ & $\begin{array}{c}-10.61 \\
(-16.75--4.46)\end{array}$ \\
\hline $\begin{array}{l}\text { 5. Message } \\
\text { conveyed by the } \\
\text { GHW is easiest to } \\
\text { understand }\end{array}$ & $\begin{array}{c}58.50 \\
(55.34-61.66)\end{array}$ & $\begin{array}{c}58.51 \\
(55.35-61.66)\end{array}$ & $\begin{array}{c}39.00 \\
(35.87-42.13)\end{array}$ & $\begin{array}{c}38.99 \\
(35.87-42.11)\end{array}$ & $\begin{array}{c}-19.50 \\
(-25.56--13.44)\end{array}$ & $\begin{array}{c}-19.51 \\
(-25.56--13.47)\end{array}$ \\
\hline $\begin{array}{l}\text { 6. Most likely to } \\
\text { lure adults into } \\
\text { using tobacco } \\
\text { products }\end{array}$ & $\begin{array}{c}-2.10 \\
(-4.18--0.02)\end{array}$ & $\begin{array}{c}-2.09 \\
(-4.15--0.03)\end{array}$ & $\begin{array}{c}-5.00 \\
(-6.77--3.23)\end{array}$ & $\begin{array}{c}-4.99 \\
(-6.75--3.24)\end{array}$ & $\begin{array}{c}-2.90 \\
(-4.45--1.35)\end{array}$ & $\begin{array}{c}-2.91 \\
(-4.45--1.37)\end{array}$ \\
\hline $\begin{array}{l}\text { 7. Most likely to } \\
\text { lure children and } \\
\text { adolescents into } \\
\text { using tobacco } \\
\text { products }\end{array}$ & $\begin{array}{c}-0.2 \\
(-2.68-2.28)\end{array}$ & $\begin{array}{c}-0.19 \\
(-2.66-2.28)\end{array}$ & $\begin{array}{c}-5.4 \\
(-7.41--3.39)\end{array}$ & $\begin{array}{c}-5.40 \\
(-7.40--3.39)\end{array}$ & $\begin{array}{c}-5.2 \\
(-7.19--3.20)\end{array}$ & $\begin{array}{c}-5.21 \\
(-7.20--3.22)\end{array}$ \\
\hline $\begin{array}{l}\text { 8. Support for } \\
\text { pack category }\end{array}$ & $\begin{array}{c}74.37 \\
(71.65-77.10)\end{array}$ & $\begin{array}{c}74.35 \\
(71.66-77.04)\end{array}$ & $\begin{array}{c}25.32 \\
(22.61-28.04)\end{array}$ & $\begin{array}{c}25.34 \\
(22.66-28.03)\end{array}$ & $\begin{array}{c}-49.05 \\
(-54.46--43.64)\end{array}$ & $\begin{array}{c}-49.01 \\
(-54.35--43.67)\end{array}$ \\
\hline
\end{tabular}

*Linear regression model adjusted for covariates that were significantly associated with the outcome. The covariates were gender (female/male), area (rural/urban), socioeconomic status (low/middle/high), age groups (13-17/18-24/25-44/ $\geq 45$ years) and tobacco use (never user/user). 01-SES excluded; 02-age group excluded; 03-gender/SES excluded; 04-gender/SES/tobacco use excluded; 05-SES/tobacco use/age group excluded; 06, 7, 8-age group and tobacco use excluded. Bold numbers indicate significance (p<0.05). 


\section{Perceptions related to the four pack categories among sub-groups}

The Supplementary file (S) summarises responses (frequencies) related to perceptions about the four pack categories in the two study areas and the association of perceptions in relation to age groups, area, gender, SES, and tobacco use status.

The $85 \%$ GHWs on conventional packs (C) and plain packs (D) were perceived to be most effective in motivating tobacco users to quit, preventing initiation by non-users and most likely to make people think of the health risks of tobacco use by over $90 \%$ of respondents. Over $90 \%$ of participants also perceived that GHWs were most noticeable and the health warning was easiest to understand on packs C and D (Supplementary Table S1). Nearly 90\% of participants believed that packs A and B were most likely and pack D was least likely to lure adults as well as adolescents into using tobacco (Supplementary Table S1).

In Delhi, a higher proportion of participants from both age groups perceived that the GHWs on category D packs were most noticeable, while in Telangana more adolescents perceived that category D pack warnings were more noticeable while the adult group perceived that pack $\mathrm{C}$ warnings were more noticeable $(\mathrm{p}<0.001)$ (Supplementary Table S2). In Telangana, more participants from the higher SES group perceived that $\mathrm{GHW}$ s on packs D (52.5\%) than on packs $\mathrm{C}(47.5 \%)$ were more noticeable (Supplementary Table S6).

Category A, the $40 \%$ old GHWs on conventional packs, was perceived to be most likely to attract adults (55\% in Delhi, $87 \%$ in Telangana) and adolescents (59\% in Delhi, $81 \%$ in Telangana) to use tobacco (Supplementary Table S1). A higher percentage of rural $(>80 \%)$ than urban $(>60 \%)$ participants perceived that packs A would be most likely to lure children and adults into using tobacco (Supplementary Table S3). In Telangana, more males (91\%) than females (83\%) perceived that packs A would be most likely to lure adults while more females (64\%) than males (53\%) perceived that they would be most likely to lure children and adolescents into using tobacco (Supplementary Table S4). In response to the question 'the GoI plans to increase the GHW from $40 \%$ to $85 \%$ which pack would you support?', $58 \%$ of participants in Delhi supported packs D compared to $74 \%$ in Telangana who supported packs C (Supplementary Table S1).

\section{DISCUSSION}

Research evidence suggests that plain packs with larger GHWs are effective in reducing pack appeal, increase noticeability of GHWs, have strong public support, have the potential to increase quitting and prevent initiation ${ }^{25-27}$.

Our study found that increasing the size of GHWs to $85 \%$ and placing them on plain packaging significantly increased the perceived noticeability of the warnings by over $40 \%$ compared with $40 \%$ size GHWs on conventional packs, when these packs were shown to the participants at the same time. While there were some variations about whether packs $\mathrm{C}$ or $\mathrm{D}$ are preferable, the study shows that increasing the size of GHW from $40 \%$ to $85 \%$ has a negative perceived effect on attracting adults and adolescents to using tobacco. It also has a positive perceived effect on motivation to quit, prevention of tobacco initiation, the seriousness of the health risks of tobacco, and understanding of the message to be conveyed by the GHWs.

This is in keeping with evidence from studies conducted in some of the high-income countries on the perceived effects of plain packaging with large GHWs on tobacco-related outcomes. A study on cigarette pack design with adult French smokers and non-smokers showed that plain packs were perceived to be more effective in convincing smokers to quit and convincing non-smokers not to initiate, compared to other branded packs ${ }^{28}$. Studies conducted in Australia ${ }^{26}$, New Zealand ${ }^{29,30}$ and the $\mathrm{UK}^{31}$ suggest that plain packaging with large GHWs reduces smoking appeal, has high public support, promotes cessation and increases attention paid to GHWs as well as recall.

The evidence so far is mainly from high-income countries and may not be generalizable to India. There is limited research on perceptions of the effects of plain packaging from LMICs ${ }^{32}$. This study from India assesses adult and adolescent perceptions of the effects of larger GHWs and plain packaging on noticeability of GHWs, the seriousness of health risks, motivation to quit, and prevention of initiation. Our results mirror findings of studies from high-income countries, thus showing that the results are pertinent 
to LMIC settings such as India, where tobacco use has been culturally unacceptable.

The study also suggests that SES, gender, age groups, the area of residence or tobacco use status did not influence the perceptions, as unadjusted and adjusted results for regression models were mostly consistent. Across states, there was evidence that all participants of Delhi supported large GHWs on plain packs while only the adolescents from Telangana believed that large GHWs on plain packs would be most effective. Participants from Delhi were primarily residents of urban settings $(87.5 \%)$ while those from Telangana were primarily residents of rural settings $(66 \%)$. Possibly, the adolescents in rural Telangana are being taught about harms of tobacco and tobacco control policies in schools and are more receptive to anti-tobacco messaging, reflected in their perceptions. Our findings are in line with those of studies conducted with adolescents in Australia that suggest that plain packs with large GHWs not only reduce the appeal of the pack $^{9,33}$ but also increase their awareness of the health consequences of tobacco use ${ }^{27}$. As adolescents represent a vulnerable target group for experimentation and initiation of tobacco use, and plain packs with large GHWs positively influence adolescents' perceptions of tobacco use prevention, as observed in our study as well as in previous studies ${ }^{27,33,34}$, it is important that this evidence-based tobacco control measure be implemented in India to protect adolescents from tobacco initiation.

\section{Strengths and limitations}

This Indian cross-sectional study, conducted in Delhi and Telangana, included men and women, urban and rural populations, and adolescent and adult age groups. This study has used well-established sampling methods, validated outcome indicators and designed tobacco packs to assess perceptions of different sizes of GHWs on conventional and plain tobacco packaging.

There are some limitations to this study. We assessed participant perceptions of the effect on outcomes. Hence, we are unable to comment on the actual effectiveness or impact of plain packaging with large GHWs on tobacco initiation, cessation, or other outcomes studied. Further, we used only four types of packs (Figure 1), which represented most likely combinations with respect to pack warnings and types relevant in the Indian policy context. These packs were shown to the participants at the same time to elicit their responses. Hence, our findings are only applicable to these four most likely combinations of packs and warnings, when shown to the participants at the same time.

Earlier studies, mostly conducted in high-income countries such as Australia ${ }^{21}$ and Canada ${ }^{22,23}$ have used smaller samples and Likert scales to assess participant perceptions. However, our communitybased study was conducted with a larger sample of 2121 participants, which included nearly $40 \%$ participants from rural Indian settings, $50 \%$ participants of low socioeconomic status, $21 \%$ illiterate population, and $12 \%$ children. There is evidence in the literature indicating that Likert scales can pose problems in low literacy populations ${ }^{35,36}$. Hence, we tried to keep the questionnaire simple for these population groups by avoiding Likert scales and asking the participants to directly choose the pack category of their choice for each question. Although the sample size was large, adolescent participants, who are most vulnerable to tobacco experimentation and initiation, were underrepresented. The majority ( $72 \%)$ of respondents had never used tobacco, consistent with the national prevalence rate. Half of the participants were classified as lower or upper-lower and only $4 \%$ as upper on the SES scale. While this could have a bearing on our results, evidence suggests that the use of most tobacco products is higher in the lower SES groups ${ }^{37}$. Further, the degree to which participants in Delhi (primarily urban) and Telangana (primarily rural) are aware of the harms of tobacco and tobacco control policies is expected to vary greatly. These differences may have been reflected in our findings.

\section{Policy implications}

This study provides research evidence in favour of larger GHWs, which is of importance in defending the tobacco industry's legal challenges against the $85 \% \mathrm{GHWs}$. Another publication from our group presents findings from a policy analysis in India that emphasizes that the logical next step for India after implementing $85 \% \mathrm{GHW}$ is plain packaging $^{38}$. Larger $\mathrm{GHW}$ sould contribute 
towards strengthening implementation of the WHO Framework Convention on Tobacco Control, recommended by the UN for the achievement of Sustainable Development Goal 3 target - reducing premature mortality due to non-communicable diseases by one-third by 2030 .

\section{CONCLUSIONS}

When four most likely combinations of pack warnings (40\% size GHW, 85\% size GHW) and pack types (conventional tobacco packs, plain packs) relevant in the Indian tobacco control policy context in the year 2016 were shown at the same time to adolescent and adult participants from two states of India, 85\% size GHWs were perceived to be more effective in increasing noticeability of warnings, motivating cessation, preventing initiation, and conveying the intended health message. Support for plain packaging was higher in Delhi and among adolescents in Telangana. Evidence-based tobacco control measures such as plain tobacco packs with large GHWs are vital to prevent experimentation and tobacco use initiation among vulnerable adolescents in LMICs such as India. Future research should explore more nuanced features of plain tobacco packs focusing on design, placement, and size of text; also field-testing of images depicting different tobacco-attributable health conditions to be used in future rotations of GHWs, as the current GHWs in India are primarily cancerfocused.

\section{REFERENCES}

1. Tata Institute of Social Sciences and Ministry of Health and Family Welfare, Government of India. Global Adult Tobacco Survey GATS 2 (GATS 2) India 201617 Report. New Delhi: Ministry of Health and Family Welfare, Government of India; 2017. https://mohfw.gov. in/newshighlights/global-adult-tobacco-survey-2-gats-2india-2016-17-report. Accessed May 10, 2019.

2. Ministry of Health and Family Welfare Government of India, World Health Organization, Centers for Disease Control and Prevention, Tata Institute of Social Sciences. GATS 2: Global Adult Tobacco Survey: Fact sheet: India 2016-17. New Delhi: Ministry of Health and Family Welfare, Government of India; 2017. https:// www.who.int/tobacco/surveillance/survey/gats/GATS_ India_2016-17_FactSheet.pdf. Accessed May 10, 2019.

3. University of Waterloo. Experts predict 1.5 million smoking deaths in India by 2020. https://uwaterloo.ca/ news/news/experts-predict-15-million-smoking-deaths- india-2020. Published September 12, 2013. Accessed October 10, 2017.

4. Arora M, Gupta VK, Nazar GP, Stigler MH, Perry CL, Reddy KS. Impact of tobacco advertisements on tobacco use among urban adolescents in India: results from a longitudinal study. Tob Control. 2012;21(3):318-324. doi:10.1136/tc.2010.040733

5. Ministry of Law and Justice, Government of India. The Cigarettes and Other Tobacco Products (Prohibition of Advertisement and Regulation of Trade and Commerce, Production, Supply and Distribution) Act, 2003. New Delhi: Government of India Press; 2003. http://legislative. gov.in/actsofparliamentfrom theyear/cigarettes-andother-tobacco-products-prohibition-advertisement-and. Accessed May 10, 2019.

6. Ministry of Health and Family Welfare. Notification - GSR 727(E) Cigarettes and Other Tobacco Products (Packaging and Labelling) Amendment Rules, 2014. http://164.100.154.238/NTCP/Acts-Rules-Regulations/ GSR-727(E).pdf. Published 2014. Accessed April 5, 2015.

7. Tobacco Labelling Resource Centre. India: Health Warnings. https://tobaccolabels.ca/countries/india/. Accessed October 15, 2018.

8. Campaign for Tobacco-free Kids. India: South East Asia Region. https://www.tobaccofreekids.org/assets/global/ pdfs/en/WL_country_India_en.pdf. Published 2015. Accessed January 5, 2018.

9. Arora M, Bhaumik S. Trade versus health: an old argument with new hope for tobacco control in India. http://blogs. bmj.com/bmjgh/2018/02/02/trade-versus-health-anold-argument-with-new-hope-for-tobacco-control-inindia/. Published February 2, 2018. Accessed February $25,2018$.

10. The Hindu. Karnataka HC strikes down rule on stringent tobacco pack warnings. http://www.thehindu.com/news/ national/karnataka-hc-strikes-down-rule-on-stringenttobacco-pack-warnings/article21725262.ece. Published December 15, 2017. Accessed January 12, 2018.

11. Hindustan Times. The Supreme Court is correct in restoring the size of pictorial warnings on cigarette packs. http://www.hindustantimes.com/editorials/the-sc-iscorrect-in-restoring-the-size-of-large-pictorial-warningson-cigarette-packs/story-elxdYPWINiXTgag0JW4JhI. html. Published January 10, 2018. Updated January 11, 2018. Accessed January 12, 2018.

12. Campaign for Tobacco-free Kids. Implementation Summary. In: Standardized or plain tobacco packaging: International developments. https://www. tobaccofreekids.org/assets/global/pdfs/en/standardized_ packaging_developments_en.pdf. Updated February 11, 2019. Accessed March 29, 2019.

13. Department of Health, Australian Government. PostImplementation Review Tobacco Plain Packaging 2016. http://ris.pmc.gov.au/sites/default/files/posts/2016/02/ Tobacco-Plain-Packaging-PIR.pdf. Accessed January 5, 
2018.

14. Australia India Institute. Report of the Australia India Institute Taskforce on Tobacco Control-Plain Packaging of Tobacco Products. Australia India Institute; 2012. https:// www.aii.unimelb.edu.au/wp-content/uploads/2019/01/ Task-force-Tobacco-Control.pdf. Accessed January 8, 2018.

15. Arora M, Tewari A, Grills N, et al. Exploring Perception of Indians about Plain Packaging of Tobacco Products: A Mixed Method Research. Front Public Health. 2013;1. doi:10.3389/fpubh.2013.00035

16. Panda B. The cigarettes and other tobacco products (prohibition of advertisement and regulation of trade and commerce, production, supply and distribution) Amendment Bill, 2014. http://164.100.47.4/billstexts/ lsbilltexts/asintroduced/1432LS.pdf. Accessed January $5,2018$.

17. Campaign for Tobacco-free Kids. Plain packaging of tobacco products toolkit: Guide 2.3. Stakeholder input/ Public consultation. https://www.tobaccofreekids.org/ microsites/plainpackaging/collecting-the-evidence/ stakeholder-input-public-consultation. Accessed November 9, 2018.

18. Office of the Registrar General \& Census Commissioner, India. Population Enumeration Data (Final Population). http://www.censusindia.gov.in/2011census/population_ enumeration.html. Accessed January 9, 2018.

19. Gajalakshmi V, Kanimozhi CV. A Survey of 24,000 Students Aged 13-15 Years in India: Global Youth Tobacco Survey 2006 and 2009. Tob Use Insights. 2010;3:23-31. doi:10.1177/1179173x1000300001

20. Bostoen K, Chalabi Z. Optimization of household survey sampling without sample frames. Int J Epidemiol. 2006;35(3):751-755. doi:10.1093/ije/dyl019

21. Parr V, Tan B, Ell P, Miller K. Market Research to Determine Effective Plain Packaging of Tobacco Products. Sydney: GfK Blue Moon; 2011.

22. CRÉATEC +. Quantitative Study of Canadian Adult Smokers: Effects of Modified Packaging Through Increasing the Size of Warnings. Canada: Health Canada; 2008.

23. CRÉATEC +. Quantitative study of Canadian youth smokers and Vulnerable non-smokers: Effects of modified packaging Through increasing the size of Warnings on cigarette packages. Canada: Health Canada; 2008.

24. Sharma R. Revised Kuppuswamy's Socioeconomic Status Scale: Explained and Updated. Indian Pediatr. 2017;54(10):867-870. doi:10.1007/s13312-017-1151-x

25. Wakefield M, Coomber K, Zacher M, Durkin S, Brennan E, Scollo M. Australian adult smokers' responses to plain packaging with larger graphic health warnings 1 year after implementation: results from a national cross-sectional tracking survey. Tob Control. 2015;24(Suppl 2):ii17-ii25. doi:10.1136/tobaccocontrol-2014-052050

26. Wakefield MA, Hayes L, Durkin S, Borland R. Introduction effects of the Australian plain packaging policy on adult smokers: a cross-sectional study. BMJ Open. 2013;3(7). doi:10.1136/bmjopen-2013-003175

27. White V, Williams T, Faulkner A, Wakefield M. Do larger graphic health warnings on standardised cigarette packs increase adolescents' cognitive processing of consumer health information and beliefs about smoking-related harms? Tob Control. 2015;24(Suppl 2):ii50-ii57. doi:10.1136/tobaccocontrol-2014-052085

28. Gallopel-Morvan K, Moodie C, Hammond D, Eker F, Beguinot E, Martinet Y. Consumer perceptions of cigarette pack design in France: a comparison of regular, limited edition and plain packaging. Tob Control. 2012;21(5):502506. doi:10.1136/tobaccocontrol-2011-050079

29. Beede P, Lawson R. The effect of plain packages on the perception of cigarette health warnings. Public Health. 1992;106(4):315-322. doi:10.1016/s0033-3506(05)80425-1

30. McCool J, Webb L, Cameron LD, Hoek J. Graphic warning labels on plain cigarette packs: will they make a difference to adolescents? Soc Sci Med. 2012;74(8):12691273. doi:10.1016/j.socscimed.2011.12.043

31. Munafo MR, Roberts N, Bauld L, Leonards U. Plain packaging increases visual attention to health warnings on cigarette packs in non-smokers and weekly smokers but not daily smokers. Addiction. 2011;106(8):1505-1510. doi:10.1111/j.1360-0443.2011.03430.x

32. Hughes N, Arora M, Grills N. Perceptions and impact of plain packaging of tobacco products in low and middle income countries, middle to upper income countries and low-income settings in high-income countries: a systematic review of the literature. BMJ Open. 2016;6(3):e010391. doi:10.1136/bmjopen-2015-010391

33. White V, Williams T, Wakefield M. Has the introduction of plain packaging with larger graphic health warnings changed adolescents' perceptions of cigarette packs and brands? Tob Control. 2015;24(Suppl 2):ii42-ii49. doi:10.1136/tobaccocontrol-2014-052084

34. Drovandi A, Teague PA, Glass B, Malau-Aduli B. A systematic review of the perceptions of adolescents on graphic health warnings and plain packaging of cigarettes. Syst Rev. 2019;8(1):25. doi:10.1186/s13643-018-0933-0

35. D'Alonzo KT. Evaluation and revision of questionnaires for use among low-literacy immigrant Latinos. Rev Lat Am Enfermagem. 2011;19:1255-1264. doi:10.1590/s0104-11692011000500025

36. Bernal H, Wooley S, Schensul JJ. The challenge of using Likert-type scales with low-literate ethnic populations. Nurs Res. 1997;46(3):179-181. doi:10.1097/00006199-199705000-00009

37. Singh A, Arora M, English DR, Mathur MR. Socioeconomic Gradients in Different Types of Tobacco Use in India: Evidence from Global Adult Tobacco Survey 2009-10. Biomed Res Int. 2015;2015. doi:10.1155/2015/837804

38. Yadav A, Nazar GP, Rawal T, Arora M, Webster P, Grills N. Plain packaging of tobacco products: the logical next 
step for tobacco control policy in India. BMJ Glob Health. 2018;3(5):e000873. doi:10.1136/bmjgh-2018-000873
ACKNOWLEDGEMENTS

We acknowledge the contribution of G.V.S. Murthy, Director, Indian Institute of Public Health (Hyderabad) for overall direction and guidance with the implementation of the study in the state of Telangana. We also acknowledge the contribution of HRIDAY, a Delhibased NGO in data collection and data management in Delhi.

\section{CONFLICTS OF INTEREST}

The authors have completed and submitted the ICMJE Form for Disclosure of Potential Conflicts of Interest and none was reported.

\section{FUNDING}

This study was funded by The Nuffield Department of Population Health, University of Oxford. No external sources other than the research team were involved: in the study design; in the collection, analysis, and interpretation of data; in the writing of the report; and in the decision to submit the paper for publication. The corresponding author had full access to all the data in the study and had final responsibility for the decision to submit the manuscript for publication.

\section{AUTHORS' CONTRIBUTIONS}

M.A., N.G., P.W., A.Y. and G.P.N. contributed to developing the concept and design of the study. G.P.N. contributed to the planning and supervising data collection and management, data analysis, interpretation of results, and drafting and revising the manuscript. V.K.G. provided technical input on data analysis and interpretation of results. T.R. assisted with the data collection, field operations, and drafting components of the manuscript. A.Y. and S.S. contributed to drafting the manuscript and providing technical inputs on tobacco control policy aspects related to plain packaging and pack warnings. N.K.K. contributed towards leading the data collection efforts, training and data management in Telangana along with contributing to drafting and reviewing manuscript components from Telangana. P.W. contributed to guiding data analysis, interpreting the results and drafting the manuscript. P.W., M.A. and N.G. contributed to revising the manuscript critically for intellectual content. All the authors approved the final version of the manuscript and are responsible for the accuracy and integrity of the work.

PROVENANCE AND PEER REVIEW

Not commissioned; externally peer reviewed. 